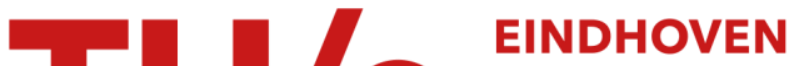 UNIVERSITY OF TECHNOLOGY
}

\section{All-optical switching and wavelength conversion based on ultrafast nonlinearities in semiconductor optical amplifiers}

\section{Citation for published version (APA):}

Dorren, H. J. S., Mishra, A. K., Yang, X., Li, Z., Ju, H., Waardt, de, H., Khoe, G. D., \& Lenstra, D. (2004). Alloptical switching and wavelength conversion based on ultrafast nonlinearities in semiconductor optical amplifiers. Journal of Applied Physics, 43(8B), 5731-5741. https://doi.org/10.1143/JJAP.43.5731

DOI:

10.1143/JJAP.43.5731

Document status and date:

Published: 01/01/2004

\section{Document Version:}

Publisher's PDF, also known as Version of Record (includes final page, issue and volume numbers)

\section{Please check the document version of this publication:}

- A submitted manuscript is the version of the article upon submission and before peer-review. There can be important differences between the submitted version and the official published version of record. People interested in the research are advised to contact the author for the final version of the publication, or visit the $\mathrm{DOI}$ to the publisher's website.

- The final author version and the galley proof are versions of the publication after peer review.

- The final published version features the final layout of the paper including the volume, issue and page numbers.

Link to publication

\section{General rights}

Copyright and moral rights for the publications made accessible in the public portal are retained by the authors and/or other copyright owners and it is a condition of accessing publications that users recognise and abide by the legal requirements associated with these rights.

- Users may download and print one copy of any publication from the public portal for the purpose of private study or research.

- You may not further distribute the material or use it for any profit-making activity or commercial gain

- You may freely distribute the URL identifying the publication in the public portal.

If the publication is distributed under the terms of Article 25fa of the Dutch Copyright Act, indicated by the "Taverne" license above, please follow below link for the End User Agreement:

www.tue.nl/taverne

Take down policy

If you believe that this document breaches copyright please contact us at:

openaccess@tue.nl

providing details and we will investigate your claim. 


\title{
All-Optical Switching and Wavelength Conversion Based on Ultrafast Nonlinearities in Semiconductor Optical Amplifiers
}

\author{
Harm J. S. DorReN ${ }^{1, *}$, Arvind K. Mishra ${ }^{1}$, Xuelin YANG $^{1}$, Zhonggui $\mathrm{Li}^{1}$, Heongkyu JU ${ }^{1}$, \\ Huug de WAARDT ${ }^{1}$, Djan KHOE ${ }^{1}$ and Daan LENSTRA ${ }^{1,2}$ \\ ${ }^{1}$ COBRA Research Institute, Eindhoven University of Technology, P.O. Box 513, 5600 MB Eindhoven, The Netherlands \\ ${ }^{2}$ Department of Physics and Astronomy, FEW, Vrije Universiteit Amsterdam, De Boelelaan 1081, 1081 HV, Amsterdam, the Netherlands \\ (Received February 18, 2004; accepted February 25, 2004; published August 25, 2004)
}

We investigate all-optical switching and wavelength conversion based on ultrafast nonlinearities in a multi-quantum-well semiconductor optical amplifier. We present a rate equation model that accounts for two-photon absorption, free-carrier absorption, self- and cross phase modulation, carrier heating, spectral, spatial hole burning and self- and cross polarization modulation. We demonstrate optical AND gate operation using a semiconductor optical amplifier placed in an asymmetric March-Zehnder interferometer and we show that the gate can be operated using $800 \mathrm{fJ}$ optical pulses with duration of $200 \mathrm{fs}$. We also investigate wavelength conversion based on ultrafast nonlinear polarization rotation. We found a conversion efficiency of $12 \mathrm{~dB}$ for control pulse energies of $10 \mathrm{pJ}$. [DOI: 10.1143/JJAP.43.5731]

KEYWORDS: optical switching, semiconductor optical amplifiers, nonlinear optics, wavelength conversion

\section{Introduction}

Digital optical techniques are expected to become increasingly important in futuristic ultrahigh capacity telecommunication networks. Optical communications systems with a capacity of hundreds of gigabits per second are commercially available today, and the capacity has been pushed above 10 terabit per second in research laboratories. The highest bit-rate per channel that has been reached is greater than 1 terabit per second. ${ }^{1)}$

For data rates greater than 40 gigabit per second, transmission systems use optical techniques since direct electronic processing is limited in speed. In optical timedomain multiplexed systems, the data at a transmission channel are demultiplexed to a lower bit-rate using a clocked optical switch. Such an optical switch can be realized by employing photo-generated changes in carrier density of semiconductor optical amplifiers (SOAs). SOAs are attractive as such a nonlinear element since they provide a high gain and exhibit a strong refractive-index change and allow photonic integration. ${ }^{2)}$

To obtain optical switching the SOA is usually placed in an interferometer. Optical switching in the picosecond regime has been shown using a variety of configurations such as the symmetric Mach-Zehnder (SMZ) interferometer, the ultrafast nonlinear interferometer (UNI) and the Terahertz optical asymmetric demultiplexer (TOAD) $)^{3-8)}$

In most applications, SOAs are operated by utilizing nonlinearities introduced by resonant optical transitions. The speed of all-optical switches based on SOA nonlinearities is determined by the carrier dynamics of the SOA. In particular, if resonant transitions are employed by using photons with an energy corresponding to the band-edge, the switching speed is restricted by the carrier lifetime, which determines the speed that carriers can be injected in the active region. Faster operation can be obtained by artificially reducing the carrier lifetime by using an additional holding beam..$^{9,10)}$ Also, it has been shown by using photons with a photon energy below the band-gap, that the SOA gain recovery time is determined by the length of the control

${ }^{*}$ Corresponding author. E-mail address: H.J.S.Dorren@tue.nl pulse due to the instantaneous two-photon-absorption (TPA) transient. ${ }^{11,12)}$ It should be noted however, the corresponding refractive index recovery is slower, since, due to the Kramers-Kronig relationship, the refractive index also depends on the gain at other wavelengths. ${ }^{12)}$

The optical switching configurations that are mentioned above, have in common that they allow operation at high repetition rates by using differential interference effects to overcome the speed limitations imposed by the carrier lifetime, while still utilizing resonant nonlinearities. It has been shown by combining switches based on nonlinear interference effects, that more sophisticated all-optical logic functionalities can be realized. In particular, proof of concept has been given for optical address recognizers, ${ }^{13-15)}$ optical bit-level synchronizers, ${ }^{16,17)}$ optical clock recovery circuits, ${ }^{18)}$ optical shift registers with inverters, ${ }^{19,20)}$ optical memories with read-write abilities, ${ }^{21-24)}$ binary half- and full adders, ${ }^{25,26)}$ pseudorandom number generators, ${ }^{27)}$ optical parity checkers ${ }^{28)}$ and optical packet switches. ${ }^{29)}$

The processing speed of the digital optical logic based on resonant optical transitions as described above is restricted to approximately $200 \mathrm{GHz}^{2)}$ The reason for this is that the SOA recovery depends on the carrier lifetime which makes that the SOA fully recovers in approximately one nanosecond. Differential operation can partly overcome this issue but at switching rates higher than $200 \mathrm{GHz},{ }^{2}$ the SOA carrier population cannot establish an equilibrium Fermi-Dirac distribution ( $5 \mathrm{ps}$ ) within one bit-time. ${ }^{30}$ )

Meanwhile, research has been carried out to investigate SOA gain and index dynamics at sub-picosecond timescales. Early experiments indicated that strong nonlinearities on sub picosecond timescales in bulk and multi-quantum-well (MQW) SOAs can take place. ${ }^{30-34)}$ These nonlinearities were soon identified to be related with non-equilibrium changes in the carrier energy distribution. ${ }^{31,35)}$ The main nonlinear processes responsible for these non-equilibrium changes were identified to be spectral hole burning, carrier heating and TPA. ${ }^{36-38)}$ Early experimental results on nonlinear index changes on sub-picosecond timescales were published in refs. 12, 39, 40 and 41. Rate-equation models that describe nonlinear carrier dynamics on sub-picosecond timescales were firstly published in refs. 36, 37, 42 and 43. Later micro- 
scopic models that describe the SOA carrier dynamics on sub-picosecond timescales have been published. ${ }^{44,45)}$

Switching experiments, employing femtosecond pulses in an ultrafast nonlinear interferometer were reported in refs. 46-49. Wavelength switching experiments employing ultrashort pulses were published in refs. 50-52. In this paper, we investigate nonlinear carrier dynamics in a MQW-SOA in the context of ultrafast all-optical logic. The nonlinear carrier dynamics of a MQW-SOA is investigated using a rate-equation model that accounts for TPA, free-carrier absorption (FCA), self- and cross phase modulation, carrier heating, spectral, spatial hole burning and self and cross polarization modulation. In particular, attention is paid to the nonlinear refractive index dynamics that is also investigated experimentally. We find that ultrashort pulses can introduce nonlinear phase changes in a MQW-SOA larger than $\pi$ radians. We also investigate an optical AND gate that consists of a single MQW SOA that is placed in an asymmetric Mach-Zehnder interferometer and wavelength conversion based on ultrafast nonlinear polarisation rotation.

\section{Theory}

A rate-equation model that describes polarization dependent nonlinear gain and index dynamics in SOAs on subpicosecond timescales is presented in ref. 53. This model takes into consideration carrier dynamics on femtosecond timescales driven by TPA and FCA, and accounts for self and cross phase modulation, carrier heating and spectral and spatial hole burning as well as self and cross polarization modulation. As shown in ref. 24, the polarization dependent gain saturation is taken into account by assuming that the polarized optical field can be decomposed into transverse electronic (TE) and transverse magnetic (TM) components that propagate "independently" through the SOA although they have indirect interaction with each other via the gain saturation. We have accounted for different TE and TM gains by assuming that these polarizations couple to different hole reservoirs. This assumption is justified by the fact that in zinc-blende structures such as GaAs and InP the optical transitions occur between an $l=0$ type conduction band state and a (degenerate) $l=1$ type valence band state. Two out of the three possible transition types are selected by the TE and TM polarizations with the two corresponding inversions. In the isotropic bulk situation, these two transitions will occur in a fully symmetric manner, but we are now interested in the case where tensile strain is built in to the bulk medium and this will cause an asymmetry between the two transition types, such that TM will be favored over TE transitions.

The incoming arbitrarily polarized electric fields is decomposed in a component parallel to the layers in the waveguide ( $x$ component, TE-mode) and a perpendicular component ( $y$ component, TM mode). These two polarization directions are along the principal axes $(\hat{x}, \hat{y})$ that diagonalise the wave propagation in the SOA. In fact, apart from their indirect interaction through the carrier dynamics in the device, these two polarizations propagate independently from each other. The total electric field is given by:

$$
\begin{aligned}
& E^{\mathrm{TE} / \mathrm{TM}}(z, t)=\left\lceil A^{\mathrm{TE}}(z, t) \hat{x}+A^{\mathrm{TM}}(z, t) \hat{y}\right\rceil e^{\mathrm{i}\left(\omega_{0} t-k_{0} z\right)}+c . c . ; \\
& S^{\mathrm{TE} / \mathrm{TM}}(z, t)=\left|A^{\mathrm{TE} / \mathrm{TM}}(z, t)\right|^{2}
\end{aligned}
$$

where $k_{0}=\left(n\left(\omega_{0}\right) / c\right) \omega_{0}, n\left(\omega_{0}\right)$ is the refractive index taken at the central frequency $\omega_{0}$ and $c$ the light velocity in vacuum, $\hat{x}$ and $\hat{y}$ are unit vectors along the $x$ and $y$ directions. The frequency $\omega_{0}$ has been chosen such that the complex pulse amplitudes $A^{\mathrm{TE} / \mathrm{TM}}(z, t)$ are slowly varying functions of $z$ and $t$. The propagation equations for the TE and TM modes are:

$$
\begin{aligned}
\left(\frac{\partial}{\partial z}+\frac{1}{v_{g}} \frac{\partial}{\partial t}\right) A^{\mathrm{TE}}(z, t)= & \left\{\frac{1}{2} \Gamma^{\mathrm{TE}}(1+\mathrm{i} \alpha) g^{\mathrm{TE}}(z, t)-\frac{1}{2} \alpha_{\mathrm{int}}\right. \\
& -\frac{1}{2} \Gamma_{2} \beta_{2}\left(1+\mathrm{i} \alpha_{2}\right)\left[S^{\mathrm{TE}}(z, t)\right. \\
& \left.+S^{\mathrm{TM}}(z, t)\right]-\frac{1}{2} \Gamma^{\mathrm{TE}} \beta_{\mathrm{c}} n_{\mathrm{c}}(z, t) \\
& \left.-\frac{1}{2} \Gamma^{\mathrm{TE}} \beta_{\mathrm{v}} n_{x}(z, t)\right\} A^{\mathrm{TE}}(z, t), \\
\left(\frac{\partial}{\partial z}+\frac{1}{v_{g}} \frac{\partial}{\partial t}\right) A^{\mathrm{TM}}(z, t)= & \left\{\frac{1}{2} \Gamma^{\mathrm{TM}}(1+\mathrm{i} \alpha) g^{\mathrm{TM}}(z, t)\right. \\
& -\frac{1}{2} \alpha_{\mathrm{int}}-\frac{1}{2} \Gamma_{2} \beta_{2}\left(1+\mathrm{i} \alpha_{2}\right) \\
& \times\left[S^{\mathrm{TE}}(z, t)+S^{\mathrm{TM}}(z, t)\right] \\
& -\frac{1}{2} \Gamma^{\mathrm{TM}} \beta_{\mathrm{c}} n_{\mathrm{c}}(z, t) \\
& \left.-\frac{1}{2} \Gamma^{\mathrm{TM}} \beta_{\mathrm{v}} n_{y}(z, t)\right\} A^{\mathrm{TM}}(z, t), \quad
\end{aligned}
$$

where the SOA parameters and their physical definitions are listed in Table I. The first term on the right-hand side of eqs. (2) and (3) represents the linear gain. $\alpha$ is the phase modulation parameter (or linewidth enhancement factor). The second term represents the TPA that is modelled by assuming that both the TE and TM modes are involved in the TPA process, where $\alpha_{2}$ is the corresponding phase modulation parameter, while the last two terms represent the FCA in the conduction and valence bands. The complex field amplitudes can be related to the intensities $S^{\mathrm{TE} / \mathrm{TM}}(z, t)$ and the phases $\phi^{\mathrm{TE} / \mathrm{TM}}(z, t)$ through the well known relationship $A^{\mathrm{TE} / \mathrm{TM}}(z, t)=\sqrt{S^{\mathrm{TE} / \mathrm{TM}}(z, t)} e^{\mathrm{i} \phi^{\mathrm{TE} / \mathrm{TM}}(z, t)}$. The gains for both modes can be expressed as:

$$
\begin{aligned}
& g^{\mathrm{TE}}(z, t)=\frac{1}{v_{g}} a^{\mathrm{TE}}\left(\omega_{0}\right)\left[n_{\mathrm{c}}(z, t)+n_{x}(z, t)-N_{0}\right] \\
& g^{\mathrm{TM}}(z, t)=\frac{1}{v_{g}} a^{\mathrm{TM}}\left(\omega_{0}\right)\left[n_{\mathrm{c}}(z, t)+n_{y}(z, t)-N_{0}\right]
\end{aligned}
$$

where $a^{\mathrm{TE} / \mathrm{TM}}\left(\omega_{0}\right)$ are the gain coefficients. Our model takes into account three bands. The numbers of electrons that are involved in the optical transition in the conduction band is $n_{\mathrm{c}}(z, t)$. We have two reservoirs of holes identified with index $x$ and $y$. Holes identified with $x$ couple with electrons through TE polarised light. The number of holes that can participate in this transition is $n_{x}(z, t)$. Similarly, holes identified with $\mathrm{y}$ couple with electrons through TM polarized light. The corresponding reservoir of holes that can participate in the optical transition is $n_{y}(z, t) . N_{0}$ is the total number of states involved in the stimulated emission. The carrier densities satisfy

$$
\begin{aligned}
\frac{\partial n_{\mathrm{c}}(z, t)}{\partial t}= & -\frac{n_{\mathrm{c}}(z, t)-\bar{n}_{\mathrm{c}}(z, t)}{\tau_{1 c}}-v_{g} g^{\mathrm{TE}}(z, t) S^{\mathrm{TE}}(z, t) \\
& -v_{g} g^{\mathrm{TM}}(z, t) S^{\mathrm{TM}}(z, t) \\
& -n_{\mathrm{c}}(z, t) \beta_{\mathrm{c}} v_{g}\left[S^{\mathrm{TE}}(z, t)+S^{\mathrm{TM}}(z, t)\right],
\end{aligned}
$$


Table I. SOA parameter definitions and their values.

\begin{tabular}{|c|c|c|c|}
\hline Parameter & Symbol & Value & Units \\
\hline Active volume & $V=L \times W \times D$ & $750 \times 2 \times 0.1$ & $\mu \mathrm{m}^{3}$ \\
\hline Confinement factor & $\Gamma_{\mathrm{TE}}, \Gamma_{\mathrm{TM}}, \Gamma_{2}$ & $0.032,0.021,0.09$ & \\
\hline FCA coefficients & $\beta_{\mathrm{c}}, \beta_{\mathrm{v}}$ & $1 \times 10^{-10}, 0$ & $\mu \mathrm{m}^{-1}$ \\
\hline Electron-hole pair lifetime & $\tau_{\mathrm{s}}$ & 1.3 & ns \\
\hline Gain coefficient & $a_{\mathrm{TE}}\left(\omega_{0}\right), a_{\mathrm{TM}}\left(\omega_{0}\right)$ & $7.0 \times 10^{-5}, 5.5 \times 10^{-5}$ & $\mu \mathrm{m}^{3} / \mathrm{ps}$ \\
\hline Group velocity & $v_{g}$ & 100 & $\mu \mathrm{m} / \mathrm{ps}$ \\
\hline Internal loss & $\alpha_{\text {int }}$ & 0.00175 & $\mu \mathrm{m}^{-1}$ \\
\hline $\begin{array}{l}\text { Optical transition energies } \\
\text { (Valence band) }\end{array}$ & $E_{\mathrm{c}}, E_{2 \mathrm{c}}$ & $0.03,0.7$ & $\mathrm{eV}$ \\
\hline $\begin{array}{l}\text { Optical transition energies } \\
\text { (Conduction band) }\end{array}$ & $E_{\mathrm{v}}, E_{2 \mathrm{v}}$ & $0.003,0.07$ & $\mathrm{eV}$ \\
\hline Carrier-carrier scattering times & $\tau_{1 c}, \tau_{1 v}$ & $0.1,0.05$ & ps \\
\hline TPA coefficient & $\beta_{2}$ & $2.5 \times 10^{-9}$ & $\mu \mathrm{m}^{2}$ \\
\hline Optical transition state density & $N_{0}$ & $1.25 \times 10^{6}$ & $\mu \mathrm{m}^{-3}$ \\
\hline
\end{tabular}

$$
\begin{aligned}
\frac{\partial n_{x}(z, t)}{\partial t}= & -\frac{n_{x}(z, t)-\bar{n}_{x}(z, t)}{\tau_{1 v}}-v_{g} g^{\mathrm{TE}}(z, t) S^{\mathrm{TE}}(z, t) \\
& -n_{x}(z, t) \beta_{\mathrm{v}} v_{g}\left[S^{\mathrm{TE}}(z, t)+S^{\mathrm{TM}}(z, t)\right] \\
\frac{\partial n_{y}(z, t)}{\partial t}= & -\frac{n_{y}(z, t)-\bar{n}_{y}(z, t)}{\tau_{1 v}}-v_{g} g^{\mathrm{TM}}(z, t) S^{\mathrm{TM}}(z, t) \\
- & n_{y}(z, t) \beta_{\mathrm{v}} v_{g}\left[S^{\mathrm{TE}}(z, t)+S^{\mathrm{TM}}(z, t)\right]
\end{aligned}
$$

The first terms on the right-hand sides of eqs. (6)-(8) describe the relaxation of the electrons and holes to their quasi-equilibrium values $\bar{n}_{i}(z, t), i \in\{c, x, y\}$ that are specified later. These relaxation processes are driven by the electronelectron and hole-hole interactions with typical timescales of 50-100 fs. The second terms describe the stimulated emission and the third term describes FCA. Before the SOA model can be completed, we need to formulate the equation for the electron-hole pair density $N(z, t)$ :

$$
\begin{aligned}
\frac{\partial N(z, t)}{\partial t}= & \frac{I}{e V}-\frac{N}{\tau_{s}}-v_{g}\left[g^{\mathrm{TE}}(z, t) S^{\mathrm{TE}}(z, t)\right. \\
& \left.+g^{\mathrm{TM}}(z, t) S^{\mathrm{TM}}(z, t)\right] \\
& +v_{g} \beta_{2}\left[S^{\mathrm{TE}}(z, t)+S^{\mathrm{TM}}(z, t)\right]^{2}
\end{aligned}
$$

where it is noted that $N(z, t)$ counts all the electron-hole pairs, including those that are not directly available for stimulated emission. The energy densities satisfy:

$$
\begin{aligned}
\frac{\partial U_{\mathrm{c}}(z, t)}{\partial t}= & \beta_{\mathrm{c}} \hbar \omega_{0} n_{\mathrm{c}}(z, t) v_{g}\left[S^{\mathrm{TE}}(z, t)+S^{\mathrm{TM}}(z, t)\right] \\
& -E_{\mathrm{c}} v_{g}\left[g^{\mathrm{TE}}(z, t) S^{\mathrm{TE}}(z, t)+g^{\mathrm{TM}}(z, t) S^{\mathrm{TM}}(z, t)\right] \\
& +E_{2 \mathrm{c}} v_{g} \beta_{2}\left[S^{\mathrm{TE}}(z, t)+S^{\mathrm{TM}}(z, t)\right]^{2} \\
& -\frac{U_{\mathrm{c}}(z, t)-\bar{U}_{\mathrm{c}}(z, t)}{\tau_{h c}}
\end{aligned}
$$

$$
\begin{aligned}
\frac{\partial U_{\mathrm{v}}(z, t)}{\partial t}= & \beta_{\mathrm{v}} \hbar \omega_{0} v_{g}\left[n_{x}(z, t)+n_{y}(z, t)\right]\left[S^{\mathrm{TE}}(z, t)+S^{\mathrm{TM}}(z, t)\right] \\
& -E_{\mathrm{v}} v_{g}\left[g^{\mathrm{TE}}(z, t) S^{\mathrm{TE}}(z, t)+g^{\mathrm{TM}}(z, t) S^{\mathrm{TM}}(z, t)\right] \\
& +E_{2 \mathrm{v}} v_{g} \beta_{2}\left[S^{\mathrm{TE}}(z, t)+S^{\mathrm{TM}}(z, t)\right]^{2}
\end{aligned}
$$

$$
-\frac{U_{\mathrm{v}}(z, t)-\bar{U}_{\mathrm{v}}(z, t)}{\tau_{h v}}
$$

where $U_{\mathrm{c}}(z, t)$ represents the energy density in the conduction band and $U_{\mathrm{v}}(z, t)$ the overall energy density in the valence band. In these equations the first terms describe the change in energy density due to FCA. The second terms describe the contribution of the stimulated emission and the third terms account for the TPA. The last term represent the relaxation to equilibrium due to carrier-phonon interactions.

The carrier density $N(z, t)$ and the energy density $U_{\mathrm{c}}(z, t)$ are needed to self-consistently calculate in each time-step the quasi-Fermi level $E_{\mathrm{fc}}(z, t)$ and temperature $T_{\mathrm{c}}(z, t)$ of the electrons, using

$$
\begin{aligned}
N(z, t) & =\frac{1}{V} \sum_{k} F\left(E_{\mathrm{fc}}(z, t), T_{\mathrm{c}}(z, t), \frac{\hbar^{2} k^{2}}{2 m_{\mathrm{c}}^{*}}\right) \\
U_{\mathrm{c}}(z, t) & =\frac{1}{V} \sum_{k} \frac{\hbar^{2} k^{2}}{2 m_{\mathrm{c}}^{*}} F\left(E_{\mathrm{fc}}(z, t), T_{\mathrm{c}}(z, t), \frac{\hbar^{2} k^{2}}{2 m_{\mathrm{c}}^{*}}\right)
\end{aligned}
$$

where $F(\mu, T, E)$ is the Fermi-Dirac distribution function defined as

$$
F(\mu, T, E)=1 /\left[1+\exp \left(\frac{E-\mu}{k T}\right)\right] .
$$

A similar procedure can be used to compute the Fermi levels and temperatures in the valence band, but one has to correct with a factor 2 since there are two sub-bands involved. The quasi-equilibrium values $\bar{n}_{i}(z, \tau)$ are given by:

$$
\begin{aligned}
& \bar{n}_{\mathrm{c}}(z, t)=N_{0} F\left(E_{\mathrm{fc}}(z, t), T_{\mathrm{c}}(z, t), \frac{\hbar^{2} k^{2}}{2 m_{\mathrm{c}}^{*}}\right) \\
& \bar{n}_{x}(z, t)=f \bar{n}_{y}(z, t)=\frac{f N_{0}}{1+f} F\left(E_{\mathrm{fv}}(z, t), T_{\mathrm{v}}(z, t), \frac{\hbar^{2} k^{2}}{2 m_{\mathrm{v}}^{*}}\right)
\end{aligned}
$$

and the quasi-equilibrium values $\bar{U}_{\mathrm{c}, \mathrm{v}}(z, t)$ are

$$
\bar{U}_{\mathrm{v}, \mathrm{c}}(z, t)=\frac{1}{V} \sum_{k} \frac{\hbar^{2} k^{2}}{2 m_{\mathrm{v}, \mathrm{c}}^{*}} F\left(E_{\mathrm{fv}, \mathrm{c}}(z, t), T_{\mathrm{L}}, \frac{\hbar^{2} k^{2}}{2 m_{\mathrm{v}, \mathrm{c}}^{*}}\right)
$$


where $T_{\mathrm{L}}$ is the lattice temperature, and $f$ is the population imbalance factor describing the gain anisotropy introduced by tensile strain in the SOA. Equation (16) describes how the equilibrium populations $\bar{n}_{x}(z, \tau)$ and $\bar{n}_{y}(z, \tau)$ are clamped to each other as a consequence of tensile strain. ${ }^{24)}$ In case of unstrained bulk material, the gain will be isotropic and $f=1$. In case of tensile strain, TM gain will be larger than TE, i.e., $f<1$. For the energy relaxation, the temperature must be taken equal to the lattice temperature $T_{\mathrm{L}}(300 \mathrm{~K})$.

We are aware of the fact that neglecting the gain and group velocity dispersion in our model for pulse propagation in a SOA limits the applicability of our model. ${ }^{54,55)}$ When the pulse duration is as short as $50-100 \mathrm{fs}$ we can no longer expect good agreement between our model and measurements. However, in view of the apparently successful application of earlier models for pulses as short as $200 \mathrm{fs}$, we expect that the present model should be applicable for pulses of the same duration, especially when the central pulse frequency coincides with the gain maximum. ${ }^{36,37,42,56)}$ Using the gain curves of the SOA that will be used in our experiments, we have estimated that for an $750-\mu \mathrm{m}$ long amplifier only minor changes occur in the pulse shape. ${ }^{57)}$ It should be remarked however, that this is no longer the case if the central pulse frequency is close to or at the zero gain region (at transparency). In this case large changes in the pulse shape take place, which could even lead to pulse break-up. ${ }^{58)}$

Moreover, we want to remark that we have modeled the phase-change by using a constant linewidth enhancement factor that is defined in the usual way. ${ }^{54,56)}$ Although this ignores dispersive effects due to strong variations in the carrier-density, such a treatment leads to result that are in agreement with experimental results.

Finally, we did not account for different group velocity dispersion coefficients for the TE and TM modes. It is shown in ref. 59 that the difference in the group velocity between the TE and TM modes is in the order of $1 \%$. We estimate that for a $500-\mu \mathrm{m}$ long SOA, the pulse distortion effects remain less than $10 \%$. This implies that for a not too long amplifier pulse pattern effects due to birefringence of the SOA are negligible.

\section{Simulation Results}

The set of eqs. (2) to (17) is solved numerically. In our simulations, the SOA length is $750 \mu \mathrm{m}$, and the active volume of $150 \mu \mathrm{m}^{3}$. We will consider optical pulses with Gaussian shape (200 fs, full width at half maximum (FWHM)) as input. The SOA pump current is $120 \mathrm{~mA}$. All other SOA parameters are listed in Table I. These parameters characterize the SOA used in the experiments in the sections that follow. The confinement factor $\Gamma^{\mathrm{TM}}$ is chosen to be $30 \%$ less than $\Gamma^{\mathrm{TM}}$ (see Table I). ${ }^{59,60)}$

It should be noted however that the SOA parameters $N_{0}$, $\Gamma^{\mathrm{TE} / \mathrm{TM}}, \alpha_{\mathrm{int}}^{\mathrm{TE} / \mathrm{TM}}, v_{g}^{\mathrm{TE} / \mathrm{TM}}$ cannot be estimated accurately. We have solved this problem by compensating the combined uncertainties in these parameter values by assigning values to $f$ and $a^{\mathrm{TE} / \mathrm{TM}}\left(\omega_{0}\right)$ in such a way that the SOA gain corresponds to typical values. In the simplest approach, one would choose $a^{\mathrm{TE}}\left(\omega_{0}\right)=a^{\mathrm{TM}}\left(\omega_{0}\right)$, which is correct in case of isotropic gain, whereas $f$ can be estimated from the measured TE and TM amplification curves by using eqs. (4),
(5), (15) and (16). In this case the polarisation dependent gain could be totally explained by the band filling effects that are represented by the factor $f$. In a somewhat more complicated approach one can assume different values for $a^{\mathrm{TE}}\left(\omega_{0}\right)$ and $a^{\mathrm{TM}}\left(\omega_{0}\right)$ as in ref. 24 for a continuous wave analysis.

The difficulties in estimating $f$ and $a^{\mathrm{TE} / \mathrm{TM}}\left(\omega_{0}\right)$ may be inherent to our modelling the SOA strain in terms of the population imbalance factor $f$. In a more accurate, but also much more complicated model, one can calculate the band structure and transition matrix elements in the presence of tensile strain and keep track of the different optical transitions involved as well as the relevant populations. This would, however, extend beyond the scope of the present approach.

The TPA coefficient has been chosen in such a way that the SOA gain saturation is in agreement with experimental results presented in ref. 58 In Fig. 1, the SOA gain is presented as a function of the pulse energy. It follows from Fig. 1 that the net amplification becomes negative for pulse energies larger than $3 \mathrm{pJ}$ in the TE mode and $1.8 \mathrm{pJ}$ in the TM mode. The curve presented in Fig. 1 is in quantitative agreement with experimental results presented in ref. 58 The net attenuation is due to the combined effects of TPA and FCA. It follows from Fig. 1 that for large pulse energies the difference in TE and TM gain almost vanishes, which can be explained by the fact that both modes equally contribute to the TPA. Thus for high energetic optical pulses the TPA terms in eqs. (4) and (6) will dominate.

In Fig. 2, the gain recovery of the TE mode is presented at different positions in the SOA for a $3.4 \mathrm{pJ}$ optical pulse. It follows that due to TPA, the pulse intensity reduces as the pulse propagates through the SOA. Deeper in the SOA, the pulse also broadens. This is a result of the TPA, which attenuates the peak intensity in combination with the gain that amplifies both wings of the pulse. Note that the SOA

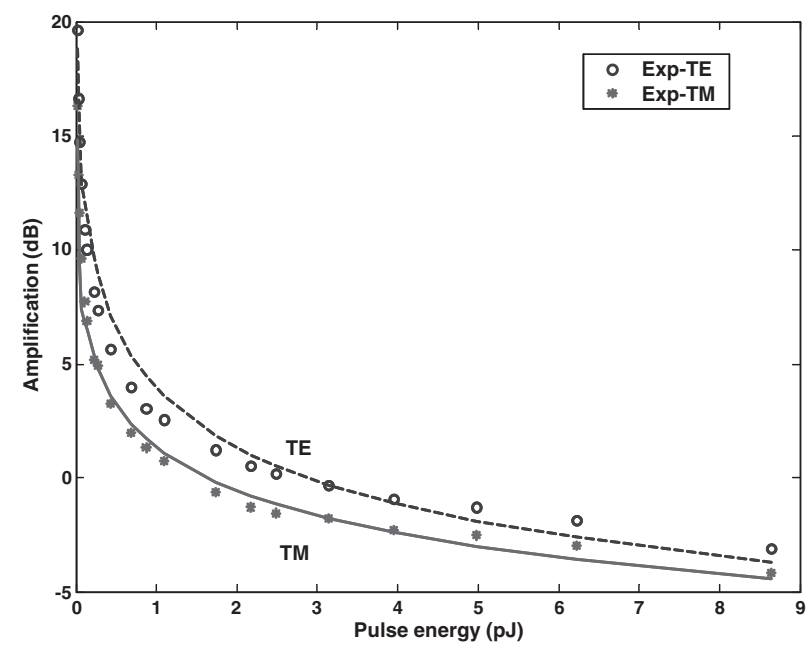

Fig. 1. Measured and computed polarization dependent amplification for the TE and TM modes as a function of the pump pulse energy. The diamond shaped points represents the measured amplification for the TE mode and the star shaped points represent the measured data for the TM mode. The solid line represents the computed result for the TE mode and the dashed line represents the computed result for the TM mode. The SOA injection current was $200 \mathrm{~mA}$ and in the computations, we used that $f=0.8$. 


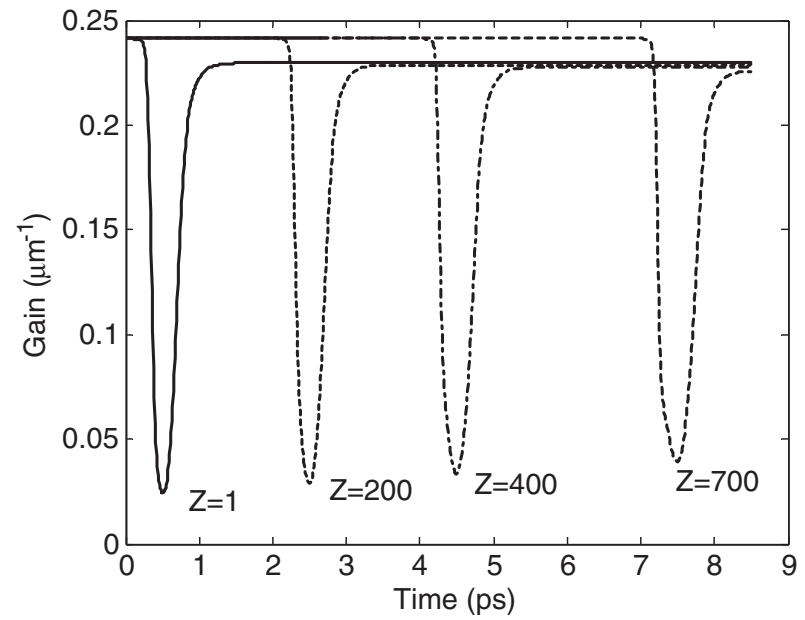

Fig. 2. Time variation of gain at different positions $z$ (in $\mu \mathrm{m}$ ) of the SOA, where the input pulse energies are $3.4 \mathrm{pJ}$ with TE mode.

gain initially recovers on a timescale of about half a picosecond.

The results that are presented so far characterize the SOA. In the following numerical experiments we investigate the feasibility of polarization switching using several pumpprobe configurations. We will consider two $200 \mathrm{fs}$ optical pulses that co-propagate through the SOA for two different situations. In the first case the pump pulse is either TE or TM polarized. In the second case the pump pulse is linearly polarized under an angle of $45 \mathrm{deg}$ with the TE and TM polarization axes.

While the pulse travels through the SOA, not only the TE and TM field component intensities will be amplified (or attenuated), but also their phase difference will change. Hence, the state of polarization changes dynamically during the propagation of the pulse through the SOA. These processes are fully described in eqs. (4) to (7).

The pump pulses have variable pulse energy. The probe pulse is linearly polarized (TE or TM) or under an angle of $45 \mathrm{deg}$ with respect to the TE and TM polarization axes. The total probe pulse energy is fixed to $0.8 \mathrm{fJ}$. This small energy guarantees that the probe pulse propagate linearly through the SOA. The delay between the pump and probe pulses is optimized to be around $5 \mathrm{fs}$ such that the latter propagates in the gain minimum introduced by the pump pulse.

In Fig. 3, the gain variations in the middle of the SOA $(z=250 \mu \mathrm{m})$ for the different polarization modes are presented. It can be observed that initially the SOA gains $g^{\mathrm{TE} / \mathrm{TM}}$ are 0.24 and $0.34 \mu \mathrm{m}^{-1}$ for the TE and TM modes respectively. As an example, in Fig. 3(a), the TE gain is presented for different pump polarizations, where the pump pulse energy is $1.7 \mathrm{pJ}$. In Fig. 3(b), a similar result is presented for the TM gain. The results show that the largest decrease in the TE gain takes place if the pump pulse is also purely TE polarized and the smallest variation in the TE gain takes place if the pump pulse is purely TM polarized. It can be noted from Fig. 3 that there is a clear gain compression induced by the strong pump pulses. After its compression, an initial gain recovery at a $0.5 \mathrm{ps}$ timescale appears, then followed by a slow recovery time, which is associated with the interband effects determined by the electron and hole

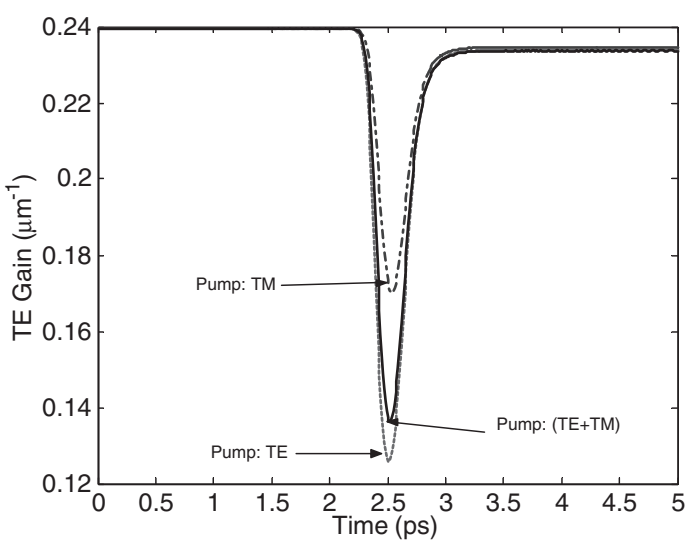

(a)

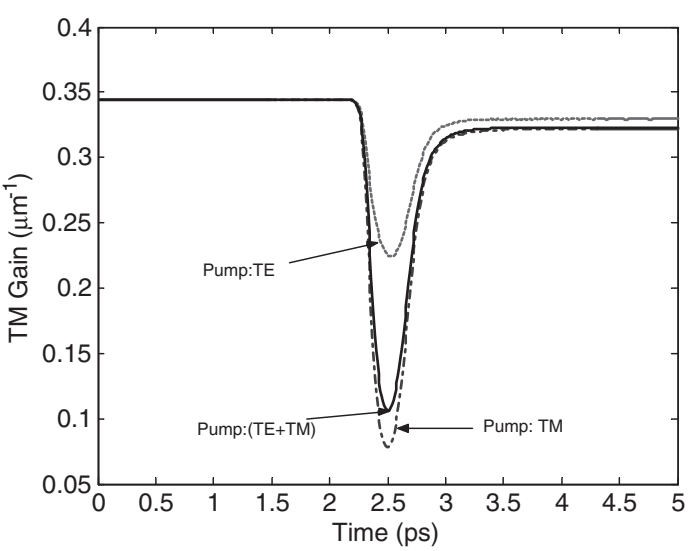

(b)

Fig. 3. Time variation of gain at the middle of SOA (at $250 \mu \mathrm{m}$ ), where the pulse energy is $1.7 \mathrm{pJ}$, and the pulse polarizations are the same as Fig. 1. In (a) the TE gain and in (b) the TM gain are shown.

recombination times (1.3ns in our cases, not shown in Fig. 3).

Because the gain temporarily decreases after the stimulated emission induced by the strong pump pulses, the probe pulse that follows will experience less amplification than when there is no pump pulse present. In Fig. 4 shows the relative amplitude differences are plotted for different pump

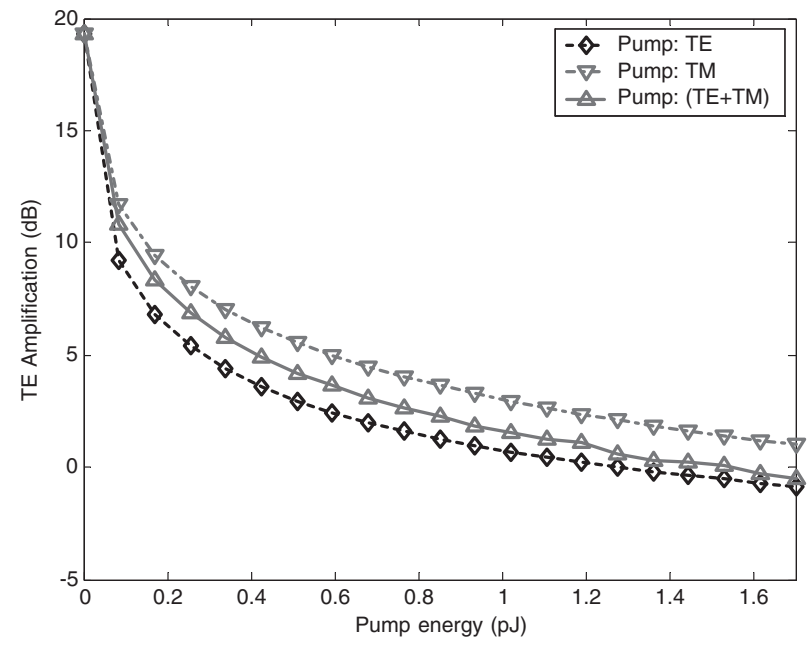

Fig. 4. Simulation of the net amplification of a $0.8 \mathrm{fJ}, 200 \mathrm{fs}$ TE polarized probe pulse as a function of the pump pulse energy. 


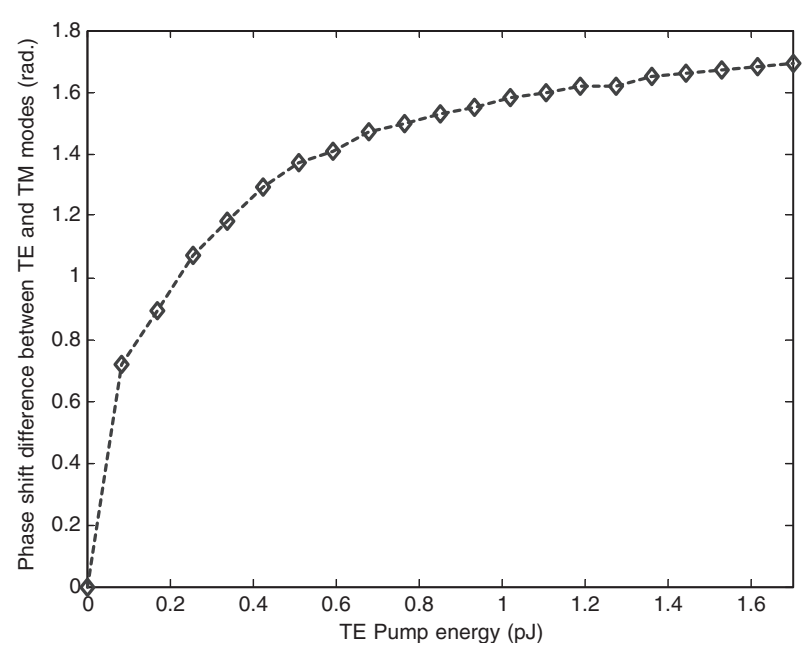

Fig. 5. Net phase shift difference $\Delta \phi$ between TE and TM modes $\left(\Delta \phi=\phi^{\mathrm{TE}}-\phi^{\mathrm{TM}}\right)$ as function of the energy of a TE polarised pump pulse.

and probe conditions. The amplification of the TE polarized probe pulse is shown as a function of pump pulse energy. It follows from Fig. 4 that, TE amplification is the smallest for TE pump, being reduced by about $20 \mathrm{~dB}$ when the pump pulse energy is $1.7 \mathrm{pJ}$. This amplification is about $0.2 \mathrm{~dB}$ larger if the pump pulse is linearly polarized under $45 \mathrm{deg}$ and another $1.5 \mathrm{~dB}$ larger if the pump is TM polarized. Similar trends can be seen if the probe pulse is TM polarized.

If the probe pulse is linearly polarized under an angle of 45 deg with respect to the TE and TM polarization axes, we can compare simultaneously the phase change for both of its TE and TM polarization components. The pump-induced phase shifts between the TE and TM components of the probe pulse as a function of the pulse energy of a TE polarized pump pulse is shown in Fig. 5. The results are calibrated with respect to the constant phase shift due to the intrinsic birefringence of the SOA when the current is $120 \mathrm{~mA}$. The largest pump-induced phase shift of a TE polarized probe pulse is about 1.8 radians for all pump polarizations at pulse energy of $1.7 \mathrm{pJ}$.

\section{Ultrafast All-Optical Switch}

In this section, we investigate an optical switch based on ultrafast SOA index nonlinearities. A schematic of our alloptical logic AND gate is shown in Fig. 6. An optical parametric oscillator (OPO) pumped with a mode-locked Ti:Sapphire laser is used to produce optical pulses that were $200 \mathrm{fs}$ FWHM in duration at a repetition rate of $75 \mathrm{MHz}$. The central wavelength of the pulses was $1520 \mathrm{~nm}$. The OPO output is firstly attenuated using a half-wave plate and a polarizer. A second half-wave plate is used to set the polarization of the laser beam to linear under $45 \mathrm{deg}$. A polarizing beam splitter is used to create a TE polarized laser beam and a TM polarized laser beam. The TM polarized laser beam forms the pump light and is fed into a variable delay line. A beam splitter divides the TE polarized laser beam into a probe beam and a reference beam. The pump and probe beams are coupled into the SOA through microscope objectives. The coupling losses are estimated

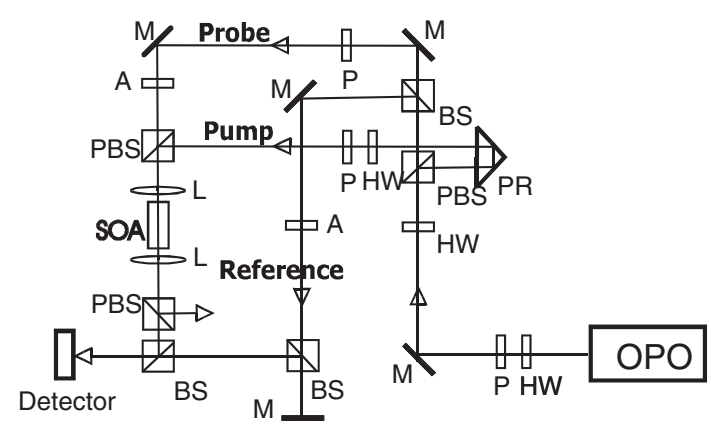

Fig. 6. Schematic of the experimental set-up, where the symbols of optical components are defined as: OPO: optical parametric oscillator, HW: half-wave plate, $\mathrm{P}$ is polarizer, $\mathrm{M}$ : mirror, $\mathrm{PBS}$ : polarizing beamsplitter, BS: beam-splitter, PR: prism, A: attenuator, L: lens.

to be $6 \mathrm{~dB}$. The pump-probe delay is controlled by the variable delay stage. The SOA used in our experiments is an InGaAsP-InGaAs MQW SOA with a central length of $750 \mu \mathrm{m}$ and a taper zone with a length of $400 \mu \mathrm{m}$ on each sides of the central part. Neutral density filters are used to control the power of the probe and reference beams. A translatable end mirror controls the delay between the reference beam and the probe beam. When the pump and probe beams have passed through the SOA, the pump light is removed by using another polarizing beam splitter. The probe and reference beams firstly interfere in the beamsplitter. The interfered light is then collimated into an optical fiber that is connected to a detector. The whole set-up is placed in a box to shield the interferometer from thermal and mechanical disturbances. An automated measurement system is employed to obtain stable measurements without any active control.

In our first experiment, the interferometer output power is measured for zero pump-probe delay. The power of the probe beam was equal to the power of the reference beam but the pump power was ten times larger. We have observed that our SOA converts about $1 \%$ of the TM polarized pump light into TE polarized light, which also interferes with the probe- and reference beams. First, we measured the maximum interferometer output (i.e. with zero time delay between probe and reference pulse) as a function of the injection current. The interferometer output power can be related to the nonlinear phase shift through the following relationship:

$$
\begin{aligned}
S_{\mathrm{det}}(\tau)= & \frac{1}{T} \int_{-T / 2}^{T / 2}\left\{P_{\mathrm{p}}(t+\tau)+P_{\mathrm{pr}}(t)+P_{\mathrm{r}}(t)\right. \\
& +2 \sqrt{P_{\mathrm{r}}(t) P_{\mathrm{p}}(t+\tau)} \cos \left[\phi_{\mathrm{r}, \mathrm{p}}-a I\right] \\
& +2 \sqrt{P_{\mathrm{p}}(t+\tau) P_{\mathrm{pr}}(t)} \cos \left[\phi_{\mathrm{p}, \mathrm{pr}}-\Delta \phi_{\mathrm{NL}}(\tau)\right] \\
& \left.+2 \sqrt{P_{\mathrm{r}}(t) P_{\mathrm{pr}}(t)} \cos \left[a I+\Delta \phi_{\mathrm{NL}}(\tau)\right]\right\} \mathrm{d} t
\end{aligned}
$$

where $S_{\mathrm{det}}(\tau)$ is the detector signal, $\tau$ the pump-probe delay, $P_{\mathrm{p}}(t+\tau)$ is the power of the polarization converted pump pulse, $P_{\mathrm{pr}}(t)$ is the power of the probe pulse and $P_{\text {ref }}(\tau)$ is the power of the reference pulse. The pump-induced nonlinear phase shift $\Delta \phi_{\mathrm{NL}}(\tau)$ is the phase difference of the probe pulse between the cases in presence and absence of a pump pulse. $\phi_{\mathrm{r}, \mathrm{p}}$ is the phase shift introduced by the optical path 


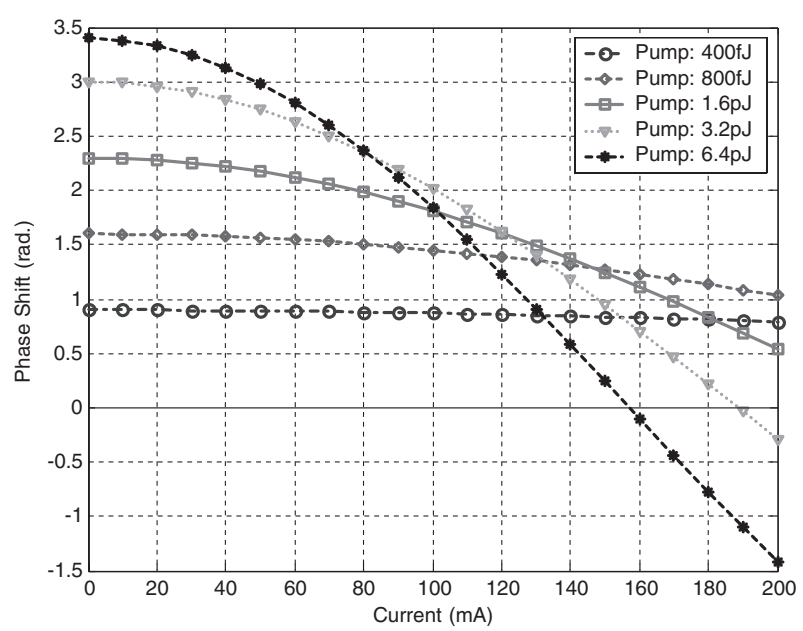

Fig. 7. Nonlinear phase shifts as a function of the injection current for various pump and probe energies when the pump-probe delay is zero.

length difference between the reference pulse and the polarization converted pump pulse. Similarly, $\phi_{\mathrm{p}, \mathrm{pr}}$ represents the phase shift introduced by the path length difference between the polarization converted pump pulse and the probe. Finally, we have observed that the phase shift induced by the SOA injection current $I$ equals to $a I$, where $a=\pi / 100 \mathrm{rad} / \mathrm{mA}$. The detector response time $T$ in eq. (18) is much larger than the pulse duration. Figure 7 shows the measured $\Delta \phi_{\mathrm{NL}}(\tau)$ as a function of the injection current. It follows that for small injection currents large positive phase shifts can be obtained. These results are in qualitative agreement with the results published in ref. 62, where it is shown that the phase shift due to TPA opposes the phase shift introduced by the gain.

The nonlinear phase shift per SOA unit length can be expressed as $2 \frac{\partial \Delta \phi_{\mathrm{NL}}(\tau)}{\partial z}=\alpha\left[g_{\mathrm{p}}(\tau)-g_{0}\right]-\alpha_{2} \beta_{2} S(\tau) .{ }^{62)}$ Here, $\alpha$ is the linewidth enhancement factor, $\beta_{2}$ the TPA coefficient, $\alpha_{2}$ the linewidth enhancement factor associated with TPA, $S(\tau)$ the photon number of the injected light, $g_{\mathrm{p}}(\tau)$ the gain in presence of a pump pulse and $g_{0}$ is the gain in absence of the pump light. The gain difference in the first contribution describes the well-known gain depletion and ultrafast recovery due to carrier cooling versus pump-probe delay time. ${ }^{37)}$ This term has an overall proportionality to the injection current $I$. For sufficiently small currents, the first contribution is positive due to dominating absorption. For a certain current, which depends on the pump pulse energy, this term turns negative due to depletion. The second term is proportional to, and has the same shape as the pump pulse. It was already concluded from the measurements in ref. 62 that $\alpha_{2}$ has a negative sign. Therefore the contribution of the second term to the phase shift is always positive. Hence, at zero current we predict the highest phase shifts, while for higher currents the phase shift decreases due to the smaller contribution of the first term.

If an ultrashort optical pump pulse is fed into an SOA that is operated at zero injection current, it will generate carriers, not only directly by absorption but also by TPA. These latter carriers are hot, but will cool down on a sub-picosecond time scale and will already lead to an extra increase of the gain within the carrier-carrier scattering time (50-100 fs), all of which leads to a positive phase shift. On the other hand, if the injection current is increased to a value above transparency, a reservoir of carriers is available in the conduction and valence bands. As soon as the optical pump pulse passes by, these carriers recombine due to stimulated emission followed by a recovery of the carrier number due to TPA and cooling. In this case, the negative gain induced contribution to the phase shift counteracts the positive instantaneous TPA induced contribution. For a specific injection current, the value of which depends on the pump pulse energy, the net phase shift vanishes. For this injection current, the phase shift due to the stimulated emission is precisely compensated by the phase shift due to TPA. ${ }^{62)}$ If the injection current is increased further the net phase shift is dominated by the stimulated emission and saturates for high injection currents.

We have also measured the contrast ratio of the optical gate using pump pulses of $800 \mathrm{fJ}$ energy. The result is shown in Fig. 8, where also the output power of the gate is plotted in the presence and absence of a pump pulse. According to Fig. 7, the best result with $800 \mathrm{fJ}$ pump pulses is expected for $I=0$. Therefore, the set up was calibrated at $I=0$ to achieve a minimum output without pump light. We measure for $I=0$ a contrast ratio of $-11 \mathrm{~dB}$. This value is limited by the residual noise in the output without pump pulse, as well as by the polarization converted pump pulse residue as discussed below eq. (18). It can be derived from eq. (18) that, given the observed noise level, if the pump pulse residue could be suppressed, the ideal contrast ratio can be as small as $-26 \mathrm{~dB}$, increasing slowly with $I$. This is indicated by the curve labeled "ideal" in Fig. 8. In this latter curve it is assumed that for each value of $I$ the system is calibrated to the minimum noise floor level in the output without pump pulse (which has not been done in the experiment). The contrast ratio can be further improved by optimizing the delay between the pump and probe pulses, the probe and reference pulse, as well as by lowering the noise floor in the output without pump pulse. In addition, we observed from the optical spectra that the output pulse did not significantly broaden after passing through the gate.

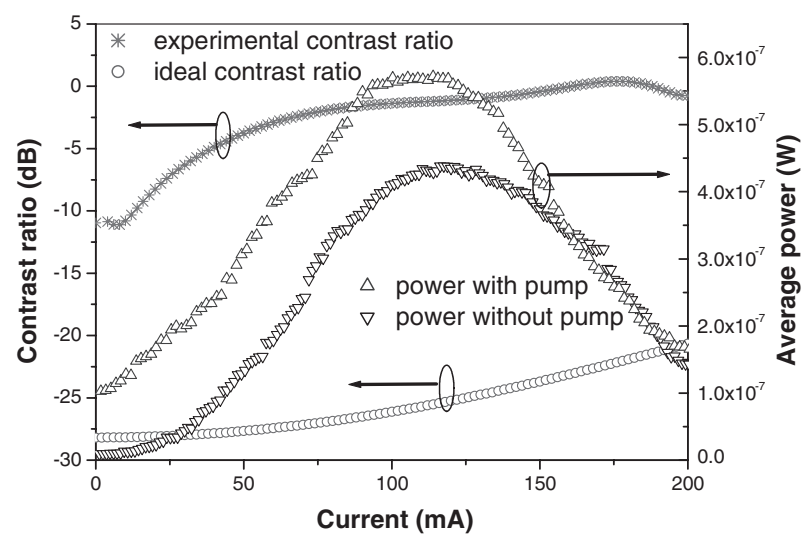

Fig. 8. Average output powers of the AND gate as a function of the injection current in presence and absence of pump light. Also shown is the contrast ratio. The pump-pulse energy is $800 \mathrm{fJ}$ while the probe (or reference) pulse energy is $80 \mathrm{fJ}$. The "ideal" curve shows the calculated contrast ratio that could be achieved if the polarization converted pump pulse residue could be removed. 


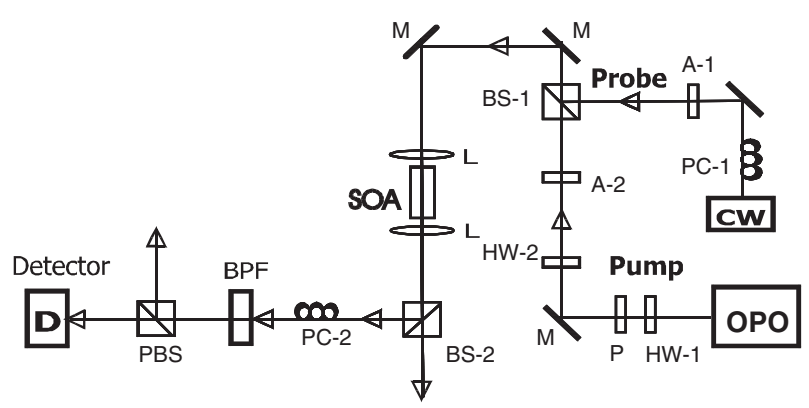

Fig. 9. Experimental implementation of the nonlinear polarization switch. OPO: optical parametric oscillator, HW: half-wave plate, P: polarizer, M: mirror, PBS: polarizing beam-splitter, BS: beam-splitter, L: lens, A: attenuator, BPF: band pass filter, PC: polarization controller, CW: Continuous wave tunable laser.

\section{Wavelength Conversion}

The scheme of our wavelength converter is shown in Fig. 9. The wavelength converter is made out of an SOA, two polarization controllers (PC-1, PC-2), two beam splitters (BS-1, BS-2), an optical band-pass filter (BPF), and a polarizing beam splitter (PBS). The OPO output is firstly attenuated using a half-wave plate (HW-1) and a polarizer. A second half-wave plate (HW-2) is used to set the polarization of the laser beam to the TE mode. A tunable laser emits a continuous wave $(\mathrm{CW})$ probe beam at wavelength $1555 \mathrm{~nm}$. The power of the probe beam is controlled by the variable attenuator (A-1) and the polarization is controlled by polarization controller PC- 1 . The pump and probe beam are combined by beamsplitter BS-1 and fed into the SOA by using microscope objectives. At the SOA output, after passing through $\mathrm{PC}-2$, the pump and probe light are separated by a bandpass filter (BPF). The BPF with a bandwidth of $1 \mathrm{~nm}$ is used to remove the pump light and to suppress the amplified spontaneous emission generated by the SOA. Finally the transmission is measured through the polarizing beamsplitter (PBS). The whole set-up is placed in a box to shield the polarization switch from thermal and mechanical disturbances.

Wavelength conversion can be realized in this set-up by setting the linear polarization of the probe beam by approximately $45 \mathrm{deg}$ with respect to the SOA layers. When a pump pulse is injected in the SOA, polarization dependent gain saturation will lead to polarization dependent index changes and thus to pump induced birefringence. The pump induced birefringence makes that the TE component of the probe beam experiences a different refractive index compared to the TM component of the probe beam which causes a rotation of the polarization of the probe beam. The rotation of the polarization is observed by measuring the transmission through the PBS. PC-2 is adjusted so that initially no light can pass through the PBS. ${ }^{63,64)}$ However, if a pumppulse is injected in the SOA the pump induced rotation of the polarization angle of the probe light, makes that some probe light can pass through the PBS. This means that at the PBS output, the pump pulse is converted to the wavelength of the probe light. ${ }^{65)}$

The discrete points in Fig. 10 show the observed PBS output for various pump pulse energies while the SOA

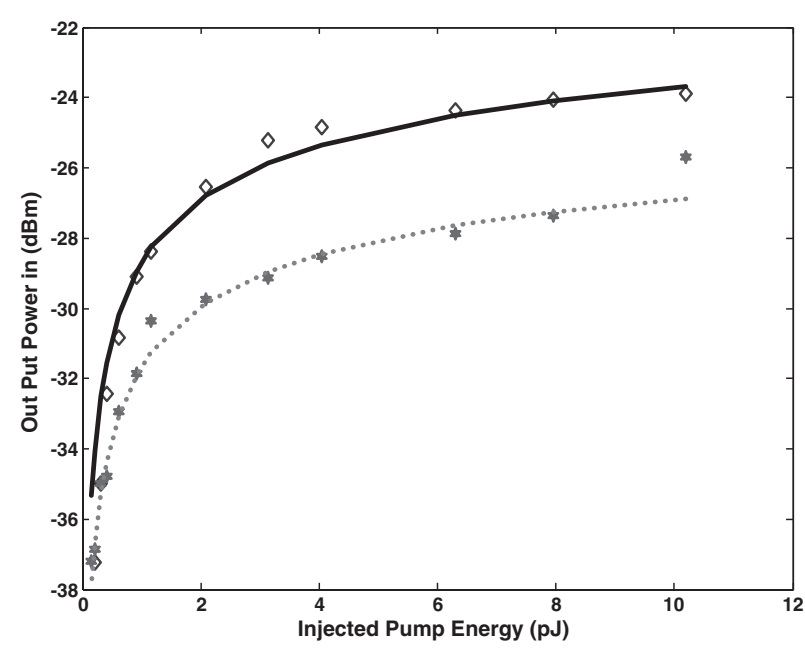

Fig. 10. Measured and computed output power of the nonlinear polarization switch as a function of pump pulse energy. The diamond shaped points show the result if the CW input power was $0 \mathrm{dBm}$ and the star shaped points represents the results if the $\mathrm{CW}$ power was $3 \mathrm{dBm}$. The SOA injection current was $200 \mathrm{~mA}$ and $f$ was 0.8 . The central wavelength of the CW light was $1555 \mathrm{~nm}$ and the central wavelength of the pump light was $1520 \mathrm{~nm}$.

injection current is $200 \mathrm{~mA}$ and the power of the $\mathrm{cw}$ probe beam is $3 \mathrm{dBm}$. The solid and dashed lines represent computed results, based on the model in $\$ 2$ and the parameters in Table I. We found a conversion efficiency larger than $12 \mathrm{~dB}$ for pulses with an energy of $10 \mathrm{pJ}$. It is clearly visible that our SOA model leads to results that are in good agreement with the experimental data. We have repeated this experiment in the case that the power of the cw beam was $0 \mathrm{dBm}$ and we found similar results.

We have also investigated wavelength conversion as a function of the injection current for different pump pulse energies. The power of the $\mathrm{cw}$ probe beam was $3 \mathrm{dBm}$. The result is shown in Fig. 11. The diamond shaped points

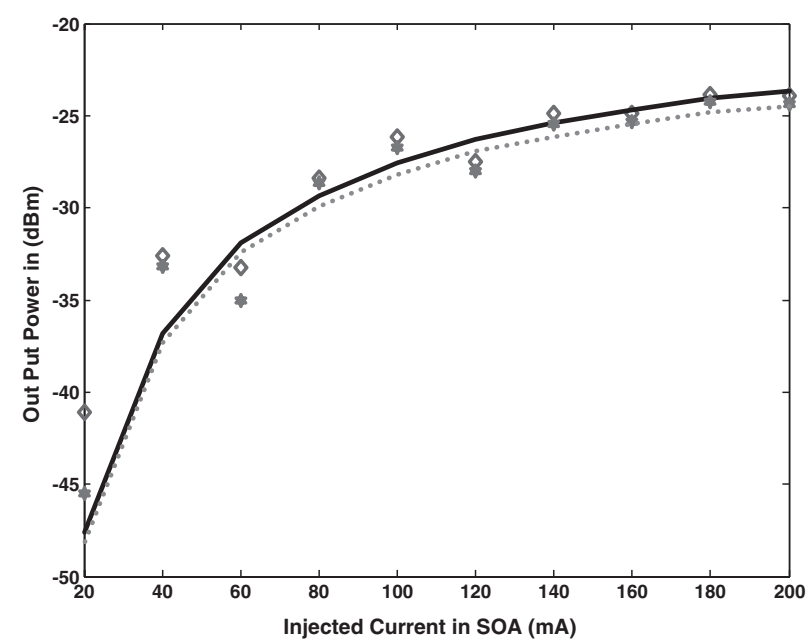

Fig. 11. Measured and computed output power of the nonlinear polarization switch as a function of the SOA injection current. The diamond shaped points shows the result if the pump pulse energy was $10 \mathrm{pJ}$ and the star shaped points represents the results if the pump pulse energy was 6.3 pJ. $f$ is as shown in Fig. 6. The central wavelength of the CW light was $1555 \mathrm{~nm}$ and central the wavelength of the pump light was $1520 \mathrm{~nm}$ and the $\mathrm{CW}$ power was $3 \mathrm{dBm}$. 
represent the results for pump energies of $10 \mathrm{pJ}$ and the star shaped points represent the results for pump pulse energies of $6.3 \mathrm{pJ}$. The solid and dashed lines represent computed results for pump pulses of $10 \mathrm{pJ}$ and $6.3 \mathrm{pJ}$ respectively, based on the model in $\S 2$ and the parameters in Table I. It is observed that the averaged converted power of the light that passes through the PBS increases as a function of current. We find our experimental result to be in good agreement with the computational result at least for the current above transparency current (50 mA).

For the wavelength conversion experiment, the average output power detected by the power meter due to polarization rotation can be formulated as:

$$
\begin{aligned}
P_{\text {out }}^{\text {Average }}= & \frac{1}{T} \int_{-T / 2}^{T / 2}\left\{S_{\mathrm{CW}}^{\mathrm{TE}}(t)+S_{\mathrm{CW}}^{\mathrm{TM}}(t)+2 \sqrt{S_{\mathrm{CW}}^{\mathrm{TE}}(t) \cdot S_{\mathrm{CW}}^{\mathrm{TM}}(t)}\right. \\
& \left.\times \cos \left(\Delta \phi_{\mathrm{NL}}(t)+\pi\right)\right\} \mathrm{d} t
\end{aligned}
$$

Where, $T$ is detector response time, $S_{\mathrm{CW}}^{\mathrm{TE}}(t)$ and $S_{\mathrm{CW}}^{\mathrm{TM}}(t)$ are the intensities of TE and TM components of the cw-light and $\Delta \phi_{\mathrm{NL}}(t)$ is the pump induced nonlinear phase difference per unit length which can be expressed as

$$
\frac{\partial \Delta \phi_{\mathrm{NL}}(t)}{\partial z}=\alpha\left[g^{\mathrm{TE}}(t)-g^{\mathrm{TM}}(t)\right]
$$

Here, $\alpha$ is the linewidth enhancement factor and $g^{\mathrm{TE}}(t)$ and $g^{\mathrm{TM}}(t)$ represents the SOA gain that accounts for TPA and FCA through the model presented in $\S 2$. Note that eq. (20) differs from its counterpart in the previous section since in eq. (20) is no direct contribution due to TPA. This is due to the fact that $\Delta \phi_{\mathrm{NL}}(t)$ represents the phase difference between the TE and TM modes. Since both modes propagate through the same SOA, the contribution to the nonlinear phase shift due to TPA is canceled out. As a result of this, the operation of a nonlinear polarization switch operated by femtosecond optical pulses differs fundamentally from a similar functionality based on nonlinear gain and index dynamics of a SOA placed in a Mach-Zehnder interferometer. ${ }^{53)}$

In Fig. 10, we observe that an increase of the pump pulse energy leads to an increase in the transmission of the probe light through the PBS. Moreover, it is clearly visible from Figs. 10 and 11 that the output power saturates for both high pump energy and high injection current. In ref. 65, it is shown that behavior is related to the SOA saturation for both high injection currents as well as for high pulse energies. Also, it is clearly visible in Figs. 10 and 11 that the wavelength converted output power is very low, which is due to the low repetition rate of the pump light. We observed a static extinction ratio larger than $12 \mathrm{~dB}$ with pump pulses having an energy of $10 \mathrm{pJ}$. This value for the energy is much higher than desired in telecommunication systems. However, it can be substantially lowered by optimizing the bandwidth of the band pass filter that is used to suppress spontaneous noise and pump pulses. Thus, it should be possible to achieve wavelength conversion operating at high repetition rates.

\section{Discussion and Future Challenges}

We have presented in this paper optical logic AND gate operation and wavelength conversion operated by ultrafast carrier dynamics in a SOA. Operation has been demonstrated by using ultrashort pulses at a low repetition rate. We have also presented a rate equation model that describes carrier dynamics in the SOA at sub-picosecond timescales. We have also shown that the operation of a nonlinear polarization switch differs on an essential point from the operation of a nonlinear optical switch based on a SOA placed in a Mach-Zehnder interferometer. This is due to the fact that both the TE and TM modes propagate through the SOA (this is in contrast to a Mach-Zehnder interferometer, were only TE modes of the probe beam propagates through the SOA ${ }^{53)}$ ) It was argued in ref. 62 that the nonlinear phase shift contains two contributions, one due to the phase shift introduced by the carrier depletion and the other due to the direct nonlinear phase shift introduced by TPA. Since in a nonlinear polarization switch both the TE and TM modes propagate through the same SOA, the direct contribution due to TPA is cancelled out. This implies that the width of the pulse that outputs the nonlinear polarization switch only depends on the nonlinear carrier dynamics in the SOA.

The largest technological challenge on the road towards the implementation of ultrafast optical logic based on SOAs nonlinearities operated at ultrahigh repetition rates, is undoubtedly related to the power consumption of these devices. Figure 1 shows that an optical pulse with an energy of $200 \mathrm{fJ}$ can suppress the SOA amplification with approximately $15 \mathrm{~dB}$. This implies that at a bit-rate of $1 \mathrm{Tbit} / \mathrm{sec}$ an average power of at least $100 \mathrm{~mW}$ is required to introduce nonlinearities that are strong enough to allow bit-wise optical switching (here it is assumed that half of the transmitted data are zero's). If switching principles based on nonlinear refractive index dynamics are employed, Fig. 7 shows that pulse energies in the order of $3 \mathrm{pJ}$ are required to introduce a phase shift of $\pi$ radians. As shown in $\S 4$, switching can be realized using pulses with much lower energy, at the expense of a high extinction ratio. In $\S 4$, we obtained an extinction ratio of $11 \mathrm{~dB}$ using control pulses with an energy of $800 \mathrm{fJ}$ in an asymmetric Mach-Zehnder interferometer. Commercially available SOAs can handle average input powers with a maximum of $50 \mathrm{~mW}$, which implies that the SOA nonlinearities are too weak to allow bitwise optical switching at ultrahigh repetition rates. We wish to remark that the coupling losses presented in this paper have been estimated conservatively, thus we expect that in reality the switching powers will be lower.

In ref. 58 results are published showing that in a $250 \mu \mathrm{m}$ long InGaAsp-InP bulk SOA, negative gain values are reached for pulses with energy of $100 \mathrm{fJ} .{ }^{58)}$ Pump and probe studies on the same device reveal that pulse energies between $460 \mathrm{fJ}$ and a few picojoules (depending on whether the SOA is in the gain or absorption regime) are required to obtain phase shifts larger than 2 radians. ${ }^{61)}$ In our device, optical pulses with energy of $800 \mathrm{fJ}$ can introduce a nonlinear phase shift of 2.3 radians.

The observed switching energies in SOAs is relatively low compared to the switching energies in passive inter-sub-band waveguides is which switching energies in the order of $10 \mathrm{pJ}$ per pulse are required to create an extinction ratio of $10 \mathrm{~dB}^{66)}$ Also, the powers required to obtain switching in an SOA seem to be an order lower compared to electroabsorption modulators. ${ }^{2,67)}$ Note that Fig. 7 shows that the 
largest nonlinear phase shifts in our SOA are obtained at zero injection current. This result also suggests that it is beneficial to carry out OTDM demuliplexing at ultrahigh bit rate by using passive devises.

A second challenge is to employ ultrafast SOA nonlinearities in more sophisticated ultrafast all-optical logic circuits. The main hurdle to be taken here is also related to the power consumption of the circuit. More complex optical logic functionalities such as optical flip-flop memories and optical pseudorandom number generators are often realized by coupling two all-optical logic gates so that the output signal of the first logic gate acts as a control signal for the second logic gate. ${ }^{23,27)}$ The optical logic gates are usually implemented by employing SOA refractive index nonlinearities in an interferometric environment (usually SOAs in a Sagnac interferometer or a Mach-Zehnder interferometer). It follows from Fig. 1, that if pulses with pulse energies greater than $2 \mathrm{pJ}$ are injected in our SOA, the output pulse energies are smaller than the input pulse energies. Figure 7 reveals that pulse with energies of $2 \mathrm{pJ}$ can introduce a nonlinear phase shift of approximately 2.7 radians. If nonlinear phase shifts greater than 2.7 radians are required, the output pulse energy of the first gate will be insufficient to control the second gate. Sophisticated integrated optical circuits consist out of active elements coupled to each other through passive elements such as splitters and filters which introduce additional losses. ${ }^{68)}$ It is therefore essential to design all-optical logic circuits that can be controlled with pulses with energies far below the critical $2 \mathrm{pJ}$ limit.

It might be possible to design optical logic gates that can be controlled with pulses of much lower energies. Interesting and promising examples include differentially operated Mach-Zehnder interferometers, or nonlinear polarization switches. ${ }^{46,69)}$ It is also believed that lower power operation can be obtained by employing quantum dot SOA. Recently the first prototype quantum dot SOAs operating at telecommunication wavelengths have been realized. ${ }^{70)}$ It should be realized however that the nonlinear carrier dynamics of quantum dot SOAs differs fundamentally from the results presented in this paper, ${ }^{71)}$ and it is not yet clear how quantum dot devices can be employed in optical logic.

\section{Acknowledgements}

This work was supported by the Netherlands Organization for Scientific Research (NWO), the Technology Foundation STW and the Ministry of Economic Affairs through respectively the NRC Photonics grant and the Innovational Research Incentives Scheme program.

1) M. Nakazawa, T. Yamamoto and K. Tamura: Electron. Lett. 36 (2000) 2027.

2) D. Cotter, R. J. Manning, K. J. Blow, A. D. Ellis, A. E. Kelly, D. Nesset, I. D. Phillips, A. J. Poustie and D. C. Rogers: Science 286 (1999) 1523.

3) J. P. Sokoloff, P. R. Prucnal, I. Glesk and M. Kane: IEEE Photon. Technol. Lett. 5 (1993) 787.

4) D. M. Patrick, A. D. Ellis, D. A. O. Davies, M. C. Tatham and G. Sherlock: Electron. Lett. 30 (1994) 341.

5) E. Jahn, N. Agrawal, M. Arbert, H. J. Ehrke, D. Franke, R. Ludwig, W. Pieper, H. G. Weber and C. M. Weinert: Electron. Lett. 31 (1995) 1857.
6) K. Tajima, S. Nakamura and Y. Sugimoto: Appl. Phys. Lett. 67 (1995) 3709.

7) N. S. Patel, K. L. Hall and K. A. Rauschenbach: Opt. Lett. 21 (1996) 1466.

8) M. Eiselt, W. Pieper and H. G. Weber: J. Lightwave Technol. 13 (1995) 2099.

9) R. J. Manning and D. A. O. Davies: Opt. Lett. 28 (1994) 1505.

10) M. T. Hill, E. Tangdiongga, H. de Waardt, G. D. Khoe and H. J. S. Dorren: Opt. Lett. 27 (2002) 1625.

11) M. Sheik-Bahae, D. C. Hutchings, D. J. Hagan and E. W. van Stryland: IEEE J. Quantum Electron. 27 (1991) 1296.

12) C. T. Hultgren, D. J. Dougherty and E. P. Ippen: Appl. Phys. Lett. 61 (1992) 2767.

13) I. Glesk, K. I. Kang and P. R. Prucnal: Electron. Lett. 30 (1994) 1322.

14) D. Cotter, J. K. Lucek, M. Shabeer, K. Smith, D. C. Rogers, D. Nesset and P. Gunning: Electron. Lett. 31 (1995) 1475.

15) N. Calabretta, H. de Waardt, G. D. Khoe and H. J. S. Dorren: IEEE Photon. Technol. Lett. 16 (2004) 1182.

16) T. J. Xia, Y. H. Kao, Y. Liang, J. W. Lou, K. H. Ahn, O. Boyraz, G. A. Nowak, A. A. Said and M. N. Islam: IEEE Photon. Technol. Lett. 11 (1999) 269.

17) N. Calabretta, Y. Liu, F. M. Huijskens, M. T. Hill, H. de Waardt, G. D. Khoe and H. J. S. Dorren: J. Lightwave Technol. 22 (2004) 372.

18) O. Kamatani and S. Kawanishi: J. Lightwave Technol. 14 (1996) 1757.

19) A. J. Poustie, R. J. Manning and K. J. Blow: Electron. Lett. 32 (1996) 1215.

20) K. L. Hall, J. P. Donnely, S. H. Groves, C. I. Fennely, R. J. Bailey and A. Napoleone: Opt. Lett. 22 (1997) 1479.

21) A. J. Poustie, K. J. Blow and R. J. Manning: Opt. Commun. 140 (1997) 184.

22) A. J. Poustie, K. J. Blow and R. J. Manning: Opt. Commun. 146 (1998) 262.

23) M. T. Hill, H. de Waardt, G. D. Khoe and H. J. S. Dorren: Microwave Opt. Technol. Lett. 31 (2001) 411.

24) H. J. S. Dorren, D. Lenstra, Y. Liu, M. T. Hill and G. D. Khoe: IEEE J. Quantum Electron. 39 (2003) 141.

25) A. J. Poustie, K. J. Blow, A. E. Kelly and R. J. Manning: Opt. Commun. 156 (1998) 22.

26) A. J. Poustie, K. J. Blow, A. E. Kelly and R. J. Manning: Opt. Commun. 168 (1999) 89.

27) A. J. Poustie, K. J. Blow, R. J. Manning and A. E. Kelly: Opt. Commun. 159 (1999) 208.

28) A. J. Poustie, K. J. Blow, A. E. Kelly and R. J. Manning: Opt. Commun. 162 (1999) 37.

29) M. T. Hill, A. Srivatsa, N. Calabretta, Y. Liu, H. de Waardt, G. D. Khoe and H. J. S. Dorren: Electron. Lett. 37 (2001) 774.

30) K. L. Hall, G. Lenz, A. M. Darwish and E. P. Ippen: Opt. Commun. 111 (1994) 589.

31) M. P. Kessler and E. P. Ippen: Appl. Phys. Lett. 51 (1987) 1765.

32) K. L. Hall, J. Mark, E. P. Ippen and G. Eisenstein: Appl. Phys. Lett. 56 (1990) 1740.

33) K. L. Hall, E. P. Ippen and G. Eisenstein: Appl. Phys. Lett. 57 (1990) 129.

34) K. L. Hall, G. Lenz, E. P. Ippen and G. Raybon: Opt. Lett. 17 (1992) 874.

35) B. N. Gomatam and A. P. DeFonso: IEEE J. Quantum Electron. 26 (1990) 1689

36) J. Mark and J. Mørk: Appl. Phys. Lett. 61 (1992) 2281.

37) J. Mørk, J. Mark and C. P. Seltzer: Appl. Phys. Lett. 64 (1994) 2206.

38) K. L. Hall, G. Lenz, E. P. Ippen, U. Koren and G. Raybon: Appl. Phys. Lett. 61 (1992) 2512.

39) C. T. Hultgren and E. P. Ippen: Appl. Phys. Lett. 59 (1991) 635.

40) K. L. Hall, Y. Lai, E. P. Ippen, G. Eisenstein and U. Koren: Appl. Phys. Lett. 57 (1990) 2888.

41) K. L. Hall, A. M. Darwish, E. P. Ippen and G. Raybon: Appl. Phys. Lett. 62 (1993) 1320.

42) J. Mørk, M. Willatzen, J. Mark, M. Svendsen and C. P. Seltzer: Proc. SPIE 2146 (1994).

43) A. Mecozzi and J. Mørk: J. Opt. Soc. Am. B 13 (1996) 2437.

44) S. Hughes, A. Knorr and S. W. Koch: J. Opt. Soc. Am. B 49 (1997) 754.

45) W. W. Chow and S. W. Koch: Semiconductor laser fundamentals (Springer Verlag, Berlin, 1999). 
46) S. Nakamura, Y. Ueno, K. Tajima, J. Sasaki, T. Sugimoto, T. Kato, T Shimoda, M. Itoh, H. Hatakeyama, T. Tamanuki and T. Sasaki: IEEE Photonics Technol. Lett. 12 (2000) 425.

47) S. Nakamura, Y. Ueno and K. Tajima: Appl. Phys. Lett. 78 (2001) 3929.

48) S. Nakamura, Y. Ueno and K. Tajima: IEEE Photonics Technol. Lett. 10 (1998) 1575.

49) H. J. S. Dorren, X. Yang, A. K. Mishra, Z. Li, H. Ju, H. de Waardt, G. D. Khoe, T. Simoyama, H. Ishikawa, H. Kawashima and T Hasama: to be published in IEEE J. Sel. Top. Quantum Electron. 10 (2004).

50) H. Kuwatsuka, T. Akiyama, B. E. Little, T. Simoyama and H. Ishikawa: Proc. ECOC 2000, Munich, Germany, 2000, Vol. 3, p. 65.

51) T. Akiyama, O. Wada, H. Kuwatsuka, T. Simoyama, Y. Nakata, K. Mukai, M. Sugawara and H. Ishikawa: Appl. Phys. Lett. 77 (2000) 1753.

52) T. Akiyama, H. Kuwatsuka, N. Hatori, Y. Nakata, H. Ebe and M. Sugawara: IEEE Photon. Technol. Lett. 14 (2002) 1139.

53) X. Yang, D. Lenstra, G. D. Khoe and H. J. S. Dorren: Opt. Commun. 223 (2003) 169.

54) S. Bischoff, A. Buxens, St. Fisher, M. Dülk, A. T. Clausen, H. N. Poulsen and J. Mørk: Opt. Quant. Electron. 33 (2003) 907.

55) M. Y. Hong, Y. H. Chang, A. Dienes, J. P. Heritage, P. J. Delfyett, S. Dijaili and F. G. Patterson: IEEE J. Sel. Top. Quantum Electron. 2 (1996) 523.

56) H. J. S. Dorren, G. D. Khoe and D. Lenstra: Opt. Commun. 205 (2002) 247.

57) M. T. Hill, H. de Waardt, G. D. Khoe and H. J. S. Dorren: IEEE J. Quantum Electron. 37 (2001) 405

58) F. Romstad, P. Borri, W. Langbein, J. Mørk and J. M. Hvam: IEEE
Photonics Technol. Lett. 12 (2000) 1674.

59) T. D. Visser, H. Blok and D. Lenstra: IEEE J. Quantum Electron. 35 (1999) 240.

60) T. D. Visser, H. Blok, B. Demeulenaere and D. Lenstra: IEEE J. Quantum Electron. 33 (1997) 1763.

61) P. Borri, W. Langbein, J. Mørk and J. M. Hvam: Opt. Commun. 169 (1999) 317.

62) H. J. S. Dorren, X. Yang, D. Lenstra, H. de Waardt, G. D. Khoe, T. Simoyama, H. Ishikawa, H. Kawashima and T. Hasama: IEEE Photonics Technol. Lett. 15 (2003) 792.

63) M. F. C. Stephens, M. Asghari, R. V. Petty and I. H. White: IEEE Photonics Technol. Lett. 9 (1997) 449.

64) H. Soto, D. Erasme and G. Guekos: IEEE Photon. Technol. Lett. 11 (1999) 970.

65) A. K. Mishra, X. Yang, D. Lenstra, G. D. Khoe and H. J. S. Dorren: to be published in IEEE J. Sel. Top. Quantum Electron. (2004).

66) T. Simoyama, H. Yoshida, J. Kasai, T. Mozume, A. V. Gopal and H. Ishikawa: IEEE Photon. Technol. Lett. 15 (2003) 1363.

67) K. Nishimura, R. Inohara, M. Tsurusawa and M. Usami: Electron. Lett. 39 (2003) 792.

68) J. H. den Besten, R. G. Broeke, M. van Geemert, J. M. M. Binsma, F. Heinrichsdorff, T. van Dongen, E. A. J. M. Bente and M. K. Smit: IEEE Photon. Technol. Lett. 15 (2003) 368.

69) H. Ju, S. Zhang, D. Lenstra, H. de Waardt, G. D. Khoe and H. J. S. Dorren: submitted to IEEE Photon. Technol. Lett. (2004).

70) T. Akiyama, K. Kawaguchi, M. Sugawara, H. Sudo, M. Ekawa, H. Ebe, A. Kuramata, K. Otsubo, K. Morito and Y. Arakawa: Proc. ECOC-ICOC 2003, Rimini, Italy, 2003, Post deadline papers, p. 80.

71) D. G. Deppe and D. L. Huffaker: Appl. Phys. Lett. 77 (2000) 3325. 